\title{
DER FALL EICHLER ZUR FRÜHGESCHICHTE DER DEUTSCHEN ARBEITERBEWEGUNG
}

Der erste Organisator grossen Stils in der sich neu konstituierenden deutschen Arbeiterbewegung der Jahre 1862-63 war ein aus Mecklenburg-Strelitz stammender Lackierer Casimir Eichler, der seit einigen Jahren in Berlin wohnte ${ }^{1}$ und bei der v. Unruhschen Fabrik zur Herstellung von Eisenbahnwagen angestellt war. Dieser Eichler stand schon im Herbst 1862 in stetiger Verbindung mit Regierungsstellen und empfing noch vor Weihnachten desselben Jahres geldliche Unterstützung von Bismarck, wie sich aus dem Nachlass Zitelmann nachweisen lässt. ${ }^{2}$ Man darf ihn daher als „Agent Bismarcks in der Arbeiterbewegung" bezeichnen, wie es im Personenverzeichnis der von Koch, Schleifstein und Höhle besorgten Neuausgabe von Mehrings Geschichte der deutschen Sozialdemokratie heisst. ${ }^{3}$ Im Index der vom Institut für Marxismus-Leninismus herausgegebenen Minutes der Londoner Konferenz der "Internationale” heisst es, Eichler habe als „agent-provocateur" in der Arbeiterbewegung gewirkt und sei als solcher später "entlarvt" worden." Beide Angaben harmonisieren, wenn wir annehmen, dass die Charakterisierung Eichlers als "Agent” sich auf den Beleg des Zitelmann-Nachlasses stützt, während die der Minutes auf Bismarcks Geständnis im Reichstag zurückgeht, Eichler habe allerdings längere Zeit der Polizei Informationen geliefert, wenn auch nicht über die Arbeiterbewegung, so jedenfalls über den Natio-

\footnotetext{
1 Nach DZA Merseburg, Rep. 77, Tit. 662, Nr 8, Band 6, Bl. 44 stammt Casimir Louis Eichler, geb. 16. März 1836, aus Templin bei Neu-Strelitz. Seit 1855 hielt er sich in Berlin auf. Dort hatte er kein Heimatsrecht, und infolge der Versammlung vom 1. September erwog die Polizei, ihm die Ausweisung anzudrohen, falls er weiter unliebsam tätig sein würde (DZA Potsdam, Rep. $30 \mathrm{C}$, Tit. 95, Sekt. 5, Nr 23, Bl. 20).

2 DZA Merseburg, Rep. 92, Zitelmann 94/I, Liquidation über Ausgaben des Dispositionsfonds 1862-1865, Fasz. 1.

3 Franz Mehring, Gesammelte Schriften, Band 1-2, Berlin 1960, dort Band 2, S. 734 .

4 The General Council of the First International 1864-1866, The London Conference 1865, Minutes, Moskau o.J., S. 450.
} 
nalverein. Bismarck ist zu seinem Geständnis durch Aussagen Bebels über die Beziehungen Bismarcks zu Lassalle gezwungen worden, über die ihm die Gräfin Hatzfeldt Einzelheiten mitgeteilt hatte. ${ }^{1}$

Bismarcks gewundene Erklärung machte die Spitzeltätigkeit Eichlers in der Arbeiterbewegung während mehrerer Jahre zur ziemlichen Gewissheit. Das ist, was Bebel in seinen Erinnerungen den „Fall Eichler" genannt hat ${ }^{2}$ - die Versuche konservativer Kreise, insbesondere Bismarcks, die Arbeiterbewegung gegen die Demokratie auszuspielen und die sogenannte „soziale Frage” für konservative politische Ziele einzuspannen. Das wäre eine, und zwar ziemlich erhebliche, Seite des Falles Eichler: die Arbeiterbewegung war jahrelang mit dem immer wiederholten Bestreben belastet, der demokrakratischen Arbeiterbewegung, besonders in der Hauptstadt, eine politisch konservative soziale Bewegung entgegenzustellen, und zwar nicht immer erfolglos. Die Verbindung von Antisemitismus mit sozialem Wohlwollen der Monarchie ist in den Jahren vor und nach Erlass des Sozialistengesetzes nicht ohne Einfluss auf die Berliner Arbeiterbewegung geblieben, wie Bernsteins Darstellung bezeugt. ${ }^{3}$ Die Vorstösse Stöckers sind bekannt, und es nimmt deshalb nicht wunder, dass man dessen Vorgänger Hermann Wagener, den Redakteur der Kreuzzeitung und den Vater des "preussischen Volksvereins” in den sechziger Jahren, dementsprechend mit Eichler in Beziehung gebracht hat. ${ }^{4}$ Eichler hat im Laufe seiner Agitation öfters hervorgehoben, dass er dem Handelsministerium gewerbliche Reformvorschläge und Anträge auf staatliche Kreditverleihung an soziale Unternehmen unterbreitet habe. Das schien ganz auf der Ebene von Wageners „Berufsrecht” des Lohnarbeiterstandes zu liegen. ${ }^{5}$

Eine Gesamtschau des Materials zum „Fall Eichler” verweist die Forschung aber in eine noch andere Richtung: sie lässt Vermutungen über Gedanken und selbst Pläne zu, die der Gesandte Bismarck schon in Paris gefasst haben mag, und die er nach dem Antritt seines Postens als Ministerpräsident weiter verfolgte. In seiner Reichs-

1 Gustav Mayer, Bismarck und Lassalle, Berlin 1928, S. $9 f$.

2 August Bebel, Aus meinem Leben, 8. Aufl., Teil I, S. 64 (,Ich wies zunächst auf den Fall Eichler hin").

${ }^{3}$ Eduard Bernstein, Die Geschichte der Berliner Arbeiterbewegung, Teil II, Berlin 1907, passim, besonders S. 58ff. (,Vergebliches Mühen des sozialen Bauernfangs").

4 Besonders deutlich Bebel, a.a.O., S. 64 und Anm. ebda.

5 Berliner Volkszeitung, Nr 89 vom 17. April 1863, und Arbeitgeber, Spalte 3009. Aber schon in der ersten Versammlung vom 25. August spricht Eichler von „Unterhandlungen mit den höchsten Staatsbehörden" (DAZ Potsdam, Rep. 30 C, a.a.O.) und am 25. Oktober von „Förderung”, die „von der Mitwirkung der Regierung" zu erwarten sei (Ebda, Bl. 27). 
tagsrede erwähnt Bismarck seinen Besuch in England im Jahre 1862, wohl im Zusammenhang mit der Weltausstellung, und die grosse Hochachtung, die ihm die Assoziationsbewegung der englischen Arbeiter eingeflösst habe. ${ }^{1}$ Er spricht nicht von den französischen sozialen Experimenten, deren sich damals der Prinz Jérôme („PlonPlon") im Interesse der Dynastie annahm und die zu der grossartig inszenierten französischen Arbeiterexpedition führten, von der die deutsche des "Nationalvereins" nur ein schwacher Abklatsch war. Das entsprach ganz Stil und Gepflogenheit im damaligen Deutschland, alles was aus Frankreich kam zu ignorieren und alles Englische als Vorbild hinzustellen. So hielten es Bismarck und der Nationalverein. Deshalb ist immer wieder von Rochdale die Rede.

Ein Polizeibericht, der von einer Agentenrolle Eichlers augenscheinlich nichts weiss, vermerkt, dass dieser bei der Rückkehr von dem vom Nationalverein organisierten Besuch der Londoner Ausstellung durch eine Gruppe von Arbeitern über Frankreich gereist sei, was als Abstecher wohl auffällig war. ${ }^{2}$ Eichler selbst bescheinigt in einem Bericht an Bismarck vom 18. Dezember 1862 den Erhalt von 90 Talern, von denen er 35 zur Tilgung der anlässlich seiner Londoner Reise gemachten Schulden verwendet habe. Es befindet sich unter den gleichen Akten auch ein leerer Umschlag, mit Blaustift „Eichler” beschrieben, der der Rechnungslage für die Monate Oktober-Dezember 1862 diente. Da die Kosten für die Reise zur Weltausstellung nach London durch ein Stipendium in Höhe von 150 Talern gedeckt waren, würde vor allem ein Abstecher nach Frankreich die Mehrausgabe erklären; ein Interesse Bismarcks an der Reise aber die Wiedervergütung. ${ }^{3}$ Uberhaupt ist die Summe von 90 Talern unverhältnismässig gross, gemessen an den Auslagen für andere Agenten. Eichlers Brief, der charakteristischerweise auf französische Verhältnisse anspielt, kann dieses Interesse erklären, indem er mit Hinweis auf die Baum-

${ }^{1}$ Die berühmte Bismarckrede vom 17. September 1878: „Der Versuch, ich weiss nicht, ob unter dem Eindruck von Lassalles Raisonnement oder unter dem Eindruck meiner eigenen Überzeugungen, die ich zum Teil in England während eines Aufenthaltes im Jahre 1862 gewonnen hatte [zum Teil in Frankreich?] - mir schien es, dass in der Herstellung von Produktivassoziationen, wie sie in England in blühendem Verhältnis existieren, die Möglichkeit lag, das Schicksal des Arbeiters zu verbessern, ihm einen wesentlichen Teil des Unternehmergewinnes zuzuwenden."

2 DZA Merseburg, Rep 77, a.a.O. Bericht vom 14. August 1862 (,,Vor etwa acht Tagen über Frankreich zurück"). Dieser Bericht klärt auch die Verwechslung mit einem Dr Ludwig Eichler auf, deren Spuren sich in vielen Darstellungen finden.

DZA Merseburg, Rep 92, Nachlass Zitelmann, Nr 16, Bl. 73, Brief Eichlers an Bismarck vom 18. Dezember 1862, und Zitelmann, Liquidation über Ausgaben des Dispositionsfonds 1862-65, Fasz. 1, Okt.-Dez. 1862, Nr 7. 
wollkrise eine ,schnelle und entscheidende Initiative der Regierung" verlangt und wegen Lösung der sozialen Frage an die Arbeiterbevölkerung zu appellieren bittet und zwar in Hinblick auf den „tiefen und gewissermassen freudigen Eindruck", den "die letzten Nachrichten aus Paris auf die hiesigen Arbeiter gemacht haben". ${ }^{1}$

Ob Eichler Bismarck in Paris oder in London gesprochen hat, lässt sich nicht feststellen, ebensowenig, was er von den bonapartistischen Bestrebungen erfahren hat; sein Besuch im kommunistischen Arbeiterverein Londons mag jedenfalls weniger Zufall sein, als bisher angenommen wurde. Es kann durchaus sein, dass Bismarck auf Eichler infolge dessen Einsatzes für die Gründung eines Arbeiterflottenvereins gestossen ist, über den sich archivalische Spuren erhalten haben. Es handelte sich dabei zwar um eine vom Nationalverein veranlasste Agitation, aber der patriotisch-preussische Akzent bei Eichlers Auftreten ist besonders aufdringlich: es könnte also dieser sonst totgeborene Flottenverein Eichler in doppelter Hinsicht nützlich gewesen sein, einmal als Empfehlung für den "Nationalverein”, wodurch er auf die Liste der Teilnehmer an der Londoner „Expedition” kam, und zum anderen wegen der Verbindung zu Konservativen, die für Bismarck Umschau hielten. ${ }^{2}$

Bismarcks „Cäsarismus” oder „Bonapartismus”, der sich aus allgemeinen Überlegungen und Charakteristiken ergibt, würde, sofern sich Eichlers Verbindungen mit dem Pariser Gesandten erhärten liessen, ein konkretes Gesicht bekommen. Bismarck hätte demnach seiner Berufung nach Berlin vorgearbeitet, sich Gedanken über die sozialen Grundlagen einer von ihm zu befolgenden Politik gemacht und gewisse Vorsätze gefasst, darunter Staatshilfe für Produktivgenossenschaften und Verbesserung der Gewerbegesetzgebung (von beiden Grundsätzen hat er sich nie losgesagt). ${ }^{3}$ Bismarck hat versucht, Bebels Darstellung seiner Beziehungen zu Eichler mit der Bemerkung zu entkräften, es sei ihm unmöglich gewesen, im September Beziehungen zu Eichler aufzunehmen, wie dieser ausgesagt hatte, da er erst Ende des Monats sein Amt angetreten habe. Diese Aussage scheint nunmehr noch fragwürdiger geworden zu sein als sie schon vorher gewesen war, aber vollkommen unwahr muss die Behauptung Bismarcks genannt werden, er habe Eichler nie bezahlt, da dieser ihm

1 Siehe den S. 349, Anm. 3 genannten Brief Eichlers.

2 DZA Potsdam, Rep 30 C, Tit. 95, Sekt. 5, Nr 28, Flottenverein der Berliner Arbeiter 1861-1863.

3 In der Bismarckrede (siehe S. 349, Anm. 1) heisst es im gleichen Absatz: „dann ebenso die Gewährung von Staatsmitteln für Produktivgenossenschaften - das ist auch eine Sache, von deren Unzweckmässigkeit ich noch heute nicht überzeugt bin." 
nie Dienste geleistet habe. Das wäre nur richtig, wenn man es mit der Wahrheit so genau nähme, dem Verhältnis Eichlers zu Bismarck das „Dienst”verhältnis abzusprechen, weil Bismarck Eichler selbstlos in seinen Bestrebungen unterstützt habe.

Eichler hat öffentlich zugegeben, dass er den höchsten Behörden Vorschläge betreffend der sozialen Frage und der Arbeiterbewegung gemacht habe und Spuren dieser Vorschläge finden sich in den Akten. ${ }^{1}$ Noch im Jahre 1865 verlangte er - allerdings vergeblich - auf Grund dieser Tätigkeit in eine beratende Kommission über die Gewerbegesetzgebung als Fachmann aufgenommen zu werden. ${ }^{2}$ Er hat Interpellationen an Parlamente und Behörden als eine der Lehren seiner Londoner Erfahrungen in Aufrufen und Rechenschaftsberichten hingestellt und hat damit auch Eindruck gemacht. Soweit könnte Eichler als Vertreter einer „staatssozialistischen” Richtung innerhalb der sich konstituierenden Arbeiterbewegung gelten und als solcher gewürdigt werden.

Selbst der folgende Satz aus dem obigen Brief an Bismarck könnte sich mit viel Wohlwollen mit eigenständigem Streben versöhnen lassen: „Ew. Excellenz sind vor allem der Mann, der berufen und befähigt ist, die jetzige Situation zu beherrschen und eine die grossen Massen des Volkes befriedigende Lösung herbeizuführen. Darin sieht aber nicht allein das Volk im eigentlichen Sinne in Preussen, sondern in ganz Europa auf Ew. Excellenz, und würde es mir zur allergrössten Freude gereichen, wenn es mir vergönnt wäre, Ew. Excellenz bei Erreichung Ihres hohen Zieles mit meinen geringen Kräften dienen zu können." Das Gleiche lässt sich aber schon nicht mehr von einem anderen Absatz sagen: „Ich erkenne mit innigster Dankbarkeit die hohe Güte und das gnädige Wohlwollen Ew. Excellenz an, werde aber auch mit allen meinen Kräften dahin streben, mich einer solchen Güte, eines solchen Wohlwollens auch fernerhin würdig zu zeigen, und hoffe ich der Regierung unseres erhabenen Königs noch grössere, erfolgreichere Dienste leisten zu können."3 Das ist einfach die Sprache eines Bedienten, der Aufträge ausführt wie sie gegeben werden.

Der „Fall Eichler” im Bebelschen Sinn wäre damit vollkommen geklärt: Da die Arbeiter Berlins mehrmals Eichlers Auftreten unmöglich gemacht haben, konnte er innerhalb der demokratischen Arbeiterbewegung nicht mehr wirken, und nachdem er seit Ende 1864 eine kleine Polizeistellung bekleidete, war ihm der Weg der

1 Siehe S. 372, Anm. 2; DZA Merseburg, Nachlass Zitelmann, a.a.O., Fasz. 6, Bl. 3; Zitelmann Nr 16, Bl. 77.

${ }^{2}$ DZA Merseburg, Rep. 120, BB I/1, Nr 12, Band 2, Bl. 15, Eingabe Eichlers an das Ministerium für Handel vom 14. August 1865.

3 Siehe S. 349, Anm. 3. 
öffentlichen Einflussnahme völlig abgeschnitten. Wie sein vergebliches Bemühen im Jahre 1865 bezeugt, war er auch für die Behörden nutzlos geworden. Der Fall wäre somit nicht nur geklärt, sondern auch erledigt - ein weiteres Beispiel für das Erfahrungsaxiom, dass die Arbeiterbewegung entweder demokratisch oder gar nicht ist.

Es wäre vielleicht noch zu beachten, dass der Fall Eichler, soweit die Annahme richtig ist, dass schon der Gesandte Bismarck aufgrund seiner französischen Beobachtungen in der Lösung der sozialen Frage einen Hebel für sein politisches Wirken sah, auch auf die Bismarckgespräche Lassalles neues Licht wirft. Der Entwurf Zitelmann für Bismarcks Schreiben an Lassalle bekäme damit besondere Bedeutung, indem Lassalle eingeladen wird, nicht nur allgemein seine Ansichten $z u$ äussern, sondern ,über die zu einer praktischen Inangriffnahme dieser Angelegenheit geeigneten Mittel und Wege" Mitteilung zu machen, womit das Gesprächsthema auf Staatskredit und ähnliche Dinge von vornherein begrenzt worden wäre. ${ }^{1}$ Solche Begrenzung, die in der letzten Fassung der Einladung nicht mehr zum Ausdruck kam, entzog Lassalles Hoffnung, Bismarck für die Abschaffung der indirekten Steuern und für die Oktroyierung des allgemeinen Stimmrechts $\mathrm{zu}$ interessieren, jeden Boden, wie tatsächlich der Gang der ersten Unterhaltung bewies, und Lassalle sich in seinem Schreiben an Bismarck vom 8. Juni selbst eingestehen musste. ${ }^{2}$ Wenn Bismarck spätestens seit dem August des Jahres 1862 und noch vor Übernahme des Ministerpostens sich auf eine bestimmte Form der Inangriffnahme der sozialen Frage festgelegt hatte, wie sein Interesse für Eichler beweist, so ist nicht nur Lassalles Illusionen jeder Boden entzogen, sondern auch all den Spekulationen, die seit der Einführung des allgemeinen Wahlrechts für den Norddeutschen Reichstag immer wieder über Inhalt und $Z$ weck der Gespräche Bismarcks mit Lassalle gemacht worden sind. Merkwürdig ist es, dass Lassalle erst im Frühjahr 1864 endgültig zu der Erkenntnis kam, dass Bismarck die soziale Frage stets unpolitisch und isoliert auffassen würde. Er hat somit für ein illusorisches politisches Konzept sich unendlich viel vergeben und seine Agitation nutzlos belastet; eingestanden hat er sich das nicht.

1 G. Mayer, Bismarck und Lassalle, S. 59 und Anm. ebda.

2 A.a.O., S. 61 (,Wenn ich letzthin Ew. Excellenz die Ansicht aussprach, dass eine zweite Unterredung unter den gegenwärtigen Umständen nutzlos bleiben müsste..."). 
Der Fall Eichler ist mit der eindeutigen Feststellung, dass Eichler Agent und Spitzel in der Arbeiterbewegung war, für die Geschichte der Arbeiterbewegung selbst noch nicht erledigt. Von den Tatsachen, die Bismarcks Geständnis aufdeckte und die durch die Einsicht in die Akten vertiefte Bedeutung bekommen, war den Zeitgenossen nichts bekannt. Man wusste nur, dass Eichler die gesetzliche Regelung der Arbeitsverhältnisse und Staatskredite für Arbeiterunternehmen verlangte, dass er eine von der liberalen Vormundschaft unabhängige Arbeiterorganisation vertrat. Erst für die Zeit nach seiner aktiven Beteiligung an der Arbeiterbewegung wusste man, dass er in Polizeidienste getreten war und zwar, weil er infolge seiner Bestrebungen seinen Arbeitsplatz verloren hatte und nirgends mehr Anstellung finden konnte. Weitergehende Behauptungen gingen auf den Meinungskampf innerhalb der Arbeiterbewegung zurück. Den Bewusstseinsstand der damaligen Berliner Arbeiterbewegung, soweit er nur auf gesicherten Tatsachen fusste, spiegelt wohl am richtigsten ein Bericht wider, den Wilhelm Liebknecht dem Generalrat der Internationalen Arbeiterassoziation in London durch Marx zukommen liess. Liebknecht war ein erfahrener und gereifter Beobachter, als er nach seiner Rückkehr aus London in die Berliner Arbeiterbewegung eintrat; er hatte die Meinungskämpfe innerhalb der Bewegung verfolgt. Der Agitation Lassalles stand er mit grosser Reserve gegenüber und wusste mehr als andere von den Bemühungen der konservativen Kreise um die Arbeiterbewegung. Aber er hatte sich mehr als andere von der Vormundschaft der liberalen Gönner der Arbeiterbewegung befreit, und war deshalb nicht bereit, sich durch Gerüchte bestimmen zu lassen. Der sich auf Eichler, beziehende Abschnitt lautet im englischen Original:

„From 1859, the time of the ,New Era', up to the end of 1862 the German workmen formed but the chorus of the ,liberal' middle-class party; at the elections they were simply, to use an American expression, the ,voting cattle' of the ,Progressists'.

The first attempt at emancipation was made at Berlin in the winter $1862 / 63$ by a workman named Eichler. He was occupied in a large Berlin factory, and by his employers he had been, together with some other workmen, sent to the London Exhibition of 1862 . In London he was accidentally present at a meeting held by the ,German Working Men's Society' (now in the International Association) in commemoration of the Paris Insurrection of June. He was struck with what he heard there, informed himself, and soon discovered that in England all that, which according to Mr. Schulze was to heal every social complaint: goose clubs, 
free competition, the right of settlement, etc., existed in full force since a long, long time, and that yet the social problem was not solved there, the fetters of labour not broken. He returned to Berlin, told his employers that Mr. Schulze was a charlatan, a humbug, and was - dismissed. He called several meetings together, explained to the workmen that in order to free themselves they must go their own way, that from the middle class they had to expect nothing and proposed a congress of the German workmen. The project met with great applause, but the intrigues of the ,Progressists', who denounced the promoter as a ,tool of the reaction' aiming at the dissolution of the unity of the great liberal party', succeeded finally in foiling it. Eichler could not get work from any employer, he was literally starved out, and, driven to the last extremity, he has since accepted a place as a telegraphist in the telegraph office of the Berlin police. It was certainly wrong in him to accept such a situation, but the principal blame no doubt falls on the middle class that systematically deprived him of every chance of living honestly."1

Liebknecht hätte vielleicht etwas mehr Achtung dem Gerücht schenken dürfen, dass Eichler ,flott" lebe, wie Bernstein in seiner Geschichte der Berliner Arbeiterbewegung betont, ${ }^{2}$ aber er mag auch das auf Hetze der Liberalen zurückgeführt haben. Es ist zwar bekannt, dass es nicht Liebknechts Veranlagung entsprach, gründliche Untersuchungen anzustellen, wo ihm der erste Eindruck ein wünschenswertes Bild bot, aber gerade auf dem Gebiet der konservativen Demagogie war er sehr argwöhnisch, weil er mit August Brass eigene Erfahrungen gemacht hatte und er Lassalle stark verdächtigte, bewusst oder unbewusst der Reaktion zu dienen. Wenn also Liebknecht als der unabhängigste und erfahrenste Beobachter Eichler für einen authentischen Arbeiterführer hielt, so ist das für das Zeitbewusstsein entscheidend wichtig.

Dem steht entgegen, dass zur gleichen Zeit nicht nur SchulzeDelitzsch, Streckfuss und Genossen, sondern ebenso die Führer der Leipziger Arbeiterbewegung, allen voran Julius Vahlteich und August Bebel, Eichler offen beschuldigten, im Dienst der Reaktion zu arbeiten,

1 The General Council, a.a.O., S. 251ff. (Report on the working class movement in Germany, written by W. Liebknecht).

Liebknechts Ausdruck ,goose-club" für Vorschussvereine klingt abfällig und scheint nicht am Platze; ,the right of settlement" meint "Niederlassungsrecht": die Übersetzung mit „Recht auf Versorgung” beruht auf einem Irrtum (siehe Die I. Internationale in Deutschland, Berlin 1964, S. 72).

${ }^{2}$ Liebknechts ,starved out" steht im Gegensatz zu Bernsteins Behauptung, die er von den liberalen Zeitungen übernommen hatte. 
Anschuldigungen, die in der sozialistischen Erinnerungsliteratur und damit auch in der Geschichtsschreibung als enthüllte Tatsachen dastehen. Die Frage ist, ob die Haltung der Leipziger dem gesunden Instinkt des Arbeiters entspricht, der Echtes von Unechtem zu unterscheiden weiss, oder ob die Einheitsfront der Liberalen mit der jungen Führung der Leipziger nicht vielmehr etwas anderes bedeutet und auf eine Problematik hinweist, die dem „Fall Eichler” noch ein anderes Gesicht gibt.

Die erste Phase der Konstituierung der deutschen Arbeiterbewegung war im Herbst 1862 erreicht worden; sie bestand in einem Wiederaufleben der Traditionen der „Arbeiterverbrüderung” der Revolutionsjahre, gründete sich auf Massenversammlungen, Kongresse und Petitionen und verlangte Unabhängigkeit und demokratische Selbständigkeit der Arbeiter. Die liberaldemokratischen Führer, insbesondere aus den Kreisen des „Nationalvereins”, hatten diese Bewegung mehr indirekt und unbewusst als direkt und bewusst gefördert: es war ihr Anliegen, die gärende Bewegung in das Bett isolierter und geschlossener Bildungsvereine zurückzudrängen. Die Hauptfront in dieser Phase lag zwischen der Kongressbewegung und dem Nationalverein. ${ }^{1}$ Der Gewinn, den die konservativen Kreise aus diesem Kampf ziehen konnten, war zunächst nur die Verlegenheit der Liberalen; die Frage, inwieweit sie aus dem Bestehen einer unabhängigen demokratischen Kongressbewegung auf die Dauer Nutzen würden ziehen können, konnte noch kaum gestellt werden. Es darf ja auch nicht vergessen werden, dass selbst in der "Arbeiterverbrüderung" gewisse zünftlerische Unterströmungen erkennbar gewesen waren, ohne dass diese die demokratische Bewegung in ein ständisches Bett hätten drängen können. Es kann auch durchaus sein, dass unter den Webern, die ein wichtiges Kontingent von Eichlers Kommitee stellten, halb zünftlerische Erinnerungen nachwirkten. ${ }^{2}$ Jedenfalls vertrat das Kommitee offiziell liberale und keine zünftlerischen Forderungen.

Die geschichtlich bedeutsame Frage wäre dementsprechend, ob die Leipziger Führer, die bei der Kaltstellung Eichlers eine Hauptrolle spielten, sich nicht durch die Liberalen täuschen liessen und trotz der nun erwiesenen Agentenrolle Eichlers der Arbeiterbewegung eine Schlappe bereitet haben.

1 Zu dieser Problematik Shlomo Na'aman, Demokratische und soziale Impulse in der Frühgeschichte der deutschen Arbeiterbewegung der Jahre 1862/63, Wiesbaden 1969, S. 18ff.

${ }^{2}$ Im Berliner Komitee waren 4 Webermeister: Nieman, Wernicke, Kalischek, Camien (DZA Potsdam, Rep. 30 C, Pol. Präs., Tit. 95, Sekt. 5, Nr 23, Bl. 24). 


\section{III}

Die Vorgänge, die der Kaltstellung Eichlers vorausgingen, seien in Kürze dargestellt. Eine an die sechzig Mann starke „Expedition” deutscher Arbeiter hatte im Sommer 1862 die Londoner Weltausstellung unter Leitung von Max Wirth, dem Herausgeber des Frankfurter Arbeitgeber, besucht. Den Grundstock bildete eine vom Nationalverein finanzierte Gruppe von 12 Arbeitern, unter denen auch Eichler war, der von der Unruh'schen Wagenfabrik deligiert war. Zweck der Expedition war, die Vorzüge des Freihandels zu studieren, und den Deutschen die englischen Errungenschaften vor Augen zu führen; einem Kongress von Arbeitervereinen sollte über Freizügigkeit und Assoziationswesen Bericht erstattet werden. Der Verlauf der Expedition war aber für Wirth persönlich und dann auch für den Nationalverein enttäuschend; es machte sich eine Opposition gegen die schlechte Organisation der Reise wie gegen die Bevormundung durch Wirth und die Honoratioren des Nationalvereins im Allgemeinen geltend, die vor allem Eichler anführte.

Wirth liess dementsprechend den Gedanken eines allgemeinen Kongresses fallen, wohingegen Eichler selbständige Anstrengungen machte, die Arbeiter Berlins für einen Arbeiterkongress zu interessieren. Er brachte Ende August und im Laufe des Monats September sechs grosse Versammlungen zustande, die die Zeitungen abgesehen von der radikalen Berliner Reform totschwiegen. Leipzig regte sich erst aufgrund der Berliner Initiative. In Berlin wie in Leipzig fusste die Bewegung auf öffentlichen Arbeiterversammlungen, aus denen auch der Kongress hervorgehen sollte: eine wichtige Neuerung, die sich allein aus den Traditionen der alten „Arbeiterverbrüderung” erklären lässt. Nach sechs Bezirksversammlungen wurde zu einer Generalversammlung Berliner Arbeiter zum 12. Oktober eingeladen, auf der Beschluss über Leipzig als Kongressort gefasst werden sollte, jedoch bereits am 9. Oktober hatte Eichler ein Gesuch um Genehmigung bei den sächsischen Behörden eingereicht. ${ }^{1}$ In Leipzig bildete sich ein Initiativkomitee erst am 30. September, das für den 2. Oktober zu einer ersten allgemeinen Arbeiterversammlung einlud.

Am 22. Oktober erschien in der Berliner Volkszeitung ein Aufruf des Berliner Komitees, von Eichler und einzelnen Komiteemitgliedern unterzeichnet, das den Kongress für den 18. November nach Leipzig einberief, und eine - später „Berliner Programm” genannte - Tagesordnung vorschlug. Auf diesen Aufruf reagierte der bekannte Berliner

1 Versammlungsbericht vom 19. Oktober (die Versammlung am 12. Oktober war wegen verspäteter Anmeldung aufgelöst worden; in ihr sollte der Aufruf des Komitees verlesen werden), DZA Potsdam, a.a.O., B1. 22. 
Volksmann Streckfuss mit einem redaktionellen Aufsatz, der über den Aufruf gesetzt wurde und das Unternehmen als unzeitgemäss verurteilte. Derselbe Streckfuss fand aber kein Wort, einen Kongress von Vertretern der Arbeiterbildungsvereine, der im Sinn von Max Wirth für den 1 . November ausgeschrieben war, abzulehnen - ein aufschlussreicher Widerspruch. ${ }^{1}$ In diesem Aufsatz fiel dann das fatale Wort von den Arbeiterversammlungen, die unbewusst der Reaktion dienten. Zwei Tage später erschien Eichler in Leipzig, um die Kongressangelegenheit zu fördern, wurde aber von dem dortigen Komitee abgewiesen, man verweigerte ihm, in einer Arbeiterversammlung aufzutreten. ${ }^{2}$

Wie kam es zu dieser scharfen und überstürzten Brüskierung Eichlers, die doch nicht nur Eichler, sondern ebenso die anderen Komiteemitglieder und hunderte von Berliner Arbeitern betraf, böses Blut machte und zum erstenmal öffentlich die Stimme laut werden liess, die liberalen Politiker gängelten die Arbeiter, eine Stimme, die sich in der Massenversammlung vom 2. November Gehör verschaffte?

An der ersten Arbeiterversammlung, die Eichler am 24. August angekündigt hatte, sollten laut Einladung ,mehrere Herren Abgeordneten" teilnehmen. Dem Bericht Bernsteins zufolge, der sich vermutlich auf Information der Berliner Reform stützte, legte dort Eichler Schulze-Delitzsch am 26. August die Frage vor, „was er von dem Projekt der Bildung eines Arbeiterbundes zum Zweck der Gründung von Arbeiterproduktionsgenossenschaften halte", wobei er sich auf Rochdale berief. Dort soll auch das böse Wort gefallen sein, die deutschen Arbeiter seien als Sklaven des Kapitals schlimmer dran als die Neger in den Südstaaten, was auf die Stimmung der Weber im Komitee zurückzuführen ist, die unter der Baumwollkrise infolge des amerikanischen Bürgerkrieges litten. ${ }^{\mathbf{3}}$ Schulze hatte als alter Demokrat die Anspielung auf die Arbeiterverbrüderung, die in dem Ausdruck „Arbeiterbund” enthalten war, gut begriffen; der Hinweis auf Rochdale, das die Freihandelsschule so eifrig propagierte,

${ }^{1}$ Berliner Volkszeitung, 1862, Nr 247. Die Zeitung hat später versucht, die auffällige Gegenüberstellung der beiden Aufsätze als „Druckschicksal” hinzustellen, was keineswegs iuberzeugend wirkt.

2 Eichlers Brief, in dem er sein Kommen ankündigt, befindet sich in dem Bestand ADAV/Hatzfeldt des IISG und ist an eine Redaktion, vermutlich die der Mitteldeutschen Volkszeitung, gerichtet. Sein Verlangen, seine „Grundsätze" darlegen $z u$ dürfen, klingt durchaus unbefangen; die Redaktion wie auch das Komitee waren aber nach dem Abdruck des Streckfuss'schen Aufsatzes von vornherein gegen Eichler eingenommen. Streckfuss hatte gute Beziehungen zu den Leipziger Demokraten, u.a. zu Dr Otto Dammer.

${ }^{3}$ Bernstein, a.a.O., Band I, S. $102 f$. 
hat ihn nicht darüber hinweggetäuscht. Sein Misstrauen war damit geweckt und der Beschluss, die Konstituierung eines „Arbeiterbundes” zu hintertreiben, gefasst, auch wenn er öffentlich davon nichts merken liess. Ein Brief Schulzes vom 26. September, wahrscheinlich an die Mitteldeutsche Volkszeitung gerichtet, gibt seiner Gegnerschaft Ausdruck; das war am Vorabend der ersten Leipziger Versammlung. ${ }^{1}$

Schulze hat auch Bennigsen über seine Gegnerschaft zu Eichler und die Kongressbewegung aufgeklärt. Als Eichler sich an Bennigsen um Unterstützung von 50 Talern aus der Kasse des Nationalvereins wandte, um den Kongress durchführen zu können, schlug dieser ab, übersandte Eichlers Schreiben an Schulze zur Information über den Mann, der "sich jetzt so breit macht und dem Ihr so wenig trauet". $\mathrm{Da}$ Eichler als Vertrauensmann des Nationalvereins an der Londoner Reise mitgewirkt hatte und die Berichterstattung über die Erfahrungen sich ganz im Rahmen der ursprünglichen Pläne hielt, war die Bitte um Unterstützung der öffentlichen Tätigkeit des Komitees am Platze und die Weigerung ungewöhnlich, denn der Nationalverein unterstützte häufig derartige Unternehmen, wenn sie den Rahmen der Partikularstaaten sprengten und damit zur nationalen Sache wurden. Die vorgeschlagene Tagesordnung des Kongresses hielt sich streng an das Programm des Nationalvereins und Eichler hatte noch zu allem Überfluss die Vorbereitung einer Propagandaschrift über den Nutzen der deutschen Einheit für den Arbeiter in Aussicht gestellt. Bennigsens guter Rat an die Arbeiter, für ihre Sache selbst aufzukommen, war beinahe eine Herausforderung. Eichler hat sich über die vielen Arbeitsausfälle beklagt, die sein weiteres Wirken in Frage stellten; Bennigsens Weitergabe dieses Briefes an Schulze am 7. November wirkte sich fast wie eine Denuntiation aus, denn wenige Tage darauf wurde Eichler aus der Wagenfabrik entlassen.

Wäre Bennigsen nicht von vornherein gegen Eichler und die Kongressidee eingenommen gewesen, hätte eine weitere Bemerkung in Eichlers Brief ihn günstig stimmen müssen. Eichler schrieb dort: „Zu meinem grössten Bedauern habe ich nun aus Leipzig vernommen, dass in einer dortigen Arbeiterversammlung so verkehrte Äusserungen gefallen sind, dass ich befürchte, die Angelegenheit kommt in eine ganz falsche Richtung, da auf dem Kongress nur gewerbliche Ange-

1 Dieser Brief Schulzes befindet sich im Bestand ADAV/Hatzfeldt des IISG; er war dem Arbeiterkomitees zur Kenntnis gekommen und wurde daher den Akten des nachmaligen Leipziger Zentralkomitees einverleibt.

${ }^{2}$ Diese Briefe in H. Oncken, „Der Nationalverein und die Anfänge der deutschen Arbeiterbewegung 1862/63", in: Grünbergs Archiv, Band II (1912), S. $120 \mathrm{ff}$. Siehe auch H. Oncken, Rudolph v. Bennigsen, Stuttgart 1910, I, S. 588. 
legenheiten dem Programm zufolge erörtert werden sollen"1; er verspricht, dass der Kongress indirekt die nationalen Belange fördern solle, aber eben nur indirekt; verhandelt solle nur die soziale Frage werden. Damit hatte Eichler seinen prinzipiellen Gegensatz zu dem Leipziger Initiativkomitee und allen voran Julius Vahlteich betont, denn in Leipzig hatte man das Verhältnis zum Nationalverein als Punkt auf die Tagesordnung gesetzt und heftige Kritik an dessen Verhalten zum Arbeiterstand geübt ${ }^{2}$ : die Leipziger wollten eine politischen Emanzipationsbewegung am linken Flügel des Nationalvereins; Eichler wollte eine reine Gewerkbewegung.

Eichler wollte also auf eine unpolitische Arbeiterorganisation heraus, die ähnlich wie Fachkongresse, die damals im Aufschwung waren, spezifische Arbeiterfragen vertreten sollte. Darauf hätten sich die Führer des Nationalvereins wohl einlassen können, wenn ihnen nicht verdächtige Zeichen des Auflebens der 48-er Erinnerungen die Sache verleidet hätten; daran waren sie in gewissem Sinne selbst schuld, denn eben hatten sie die alte Reichsverfassung mit Grundrechten und demokratischem Wahlstatut als Programmpunkt angenommen. ${ }^{3}$ Die Berliner Führung der Fortschrittler war aber besonders vorsichtig; sie stand im Verfassungskonflikt und war entschlossen, ihn rein parlamentarisch und ohne Massenbeteiligung durchzukämpfen. Jede Massenaufregung und Massenorganisation musste ihnen da unbequem sein. Daher kam es, dass den Berliner Fortschrittlern die Leipziger weniger gefährlich schienen als Eichler, erstens weil sie Leipziger und keine Berliner waren, und dann, weil sie politisch dachten und daher dem Argument zugänglich waren, der Verfassungskampf verlange zeitweiliges Zurückhalten, um der Reaktion keine Vorwände zu bieten.

\section{IV}

Der Aufruf des Berliner Zentralkomitees an sämtliche Arbeiter Deutschlands „Arbeiter! Deutsche Brüder!” war schon in der Art der Ansprache unverfänglich nationalvereinlerisch. Der Stil ist farbenund phrasenreich, aber keineswegs besonders "verschwommen",

1 Damit sind die "Grundsätze" erklärt, die Eichler den Leipzigern vortragen wollte. Mehrings mehrfach geäusserte Behauptung, der auch Bernstein folgt, dass damit Staatskredit durch Bismarck gemeint sei, stützt sich auf Bebel (Mehring, Geschichte der deutschen Sozialdemokratie, II, S. 22).

${ }^{2}$ Der Aufruf des Leipziger Arbeiterkomitees in der Mitteldeutschen Volkszeitung vom 30. September 1862 verspricht den „von Berlin aus beabsichtigten Arbeiterkongress sowie das Verhältnis zum Nationalverein" zu erörtern.

${ }^{3}$ Generalversammlung des Nationalvereins zu Koburg am 6.-7. Oktober 1862. 
wie Bernstein rügt.1 „Die Sonne eines neuen Lebens” (die „Neue Ära”) habe alle Klassen der Bevölkerung zu eigener Tätigkeit angeregt (Schulzes oft wiederholte Phrase von der Volksinitiative, die das Kennzeichnen der neuen Zeit sei), aber die Arbeiter allein hätten sich auf andere verlassen, und zum Dank sei nun der Zünftlertag in Weimar gekommen (gegen dessen Beschlüsse eine Nürnberger Tagung am 1. November protestieren sollte), der Gewerbefreiheit und Freizügigkeit begraben wolle. Dann geht der Aufruf auf die neueste Entwicklung ein: „Was soll daraus werden, wenn der neue französische Handelsvertrag in Kraft tritt und unsere Industrie der Konkurrenz eines Landes aussetzt, in dem alle die grossen wirtschaftlichen Errungenschaften längst anerkannt und durchgeführt sind? „Weiter wird angeregt, zum Zweck gewerblicher Reformen und der Bekämpfung reaktionärer Bestrebungen in Leipzig zu einem allgemeinen Arbeiterkongress zusammenzutreten, damit "derselbe Zeugnis gebe von den Wünschen und Bedürfnissen des deutschen Arbeiterstandes". Besondere Bedeutung sollte der Berichterstattung aller Besucher der Londoner Industrieausstellung zukommen, da diese sich mit den englischen Arbeiter-Einrichtungen vertraut gemacht hätten.

Das „Programm der auf dem Kongress zur Erörterung kommenden Vorlagen” lautete: „Die Einführung der Gewerbefreiheit durch ganz Deutschland. Die Einführung der Freizügigkeit durch ganz Deutschland. Die Beratung und Feststellung von Grundstatuten für Assoziationen und Invalidenkassen für alte Arbeiter. Die Arrangierung einer Welt-Industrieausstellung zu Berlin in den nächsten Jahren. Gewerbliche Mitteilungen der nach der Londoner Ausstellung gewesenen Arbeiter."2 Die Delegierten wurden ersucht, über die Punkte der Tagesordnung nach entsprechender Beratung Vorschläge beim Komitee $z u$ machen und Resolutionen einzureichen, und ihre Wahl den Redaktionen der Berliner liberalen Zeitungen anzuzeigen.

Alle Redaktionen deutscher Zeitungen wurden gebeten, den Aufruf abzudrucken. Wollte die Nationalpartei den Arbeitern das zugestehen, was sie für Juristen, Naturwissenschaftler und Volkswirte für passend fand, so war dieser Aufruf ganz am Platz, er reihte sich den anderen „Volksinitiativen” stilgemäss und inhaltlich makellos an.

Die Antwort gab der geharnischte Protest von Adolf Streckfuss. ${ }^{3}$

1 Teilweiser Abdruck bei Bernstein, a.a.O., S. 104. Durch Auslassung einiger sachlich wichtiger Stellen wird der Eindruck der Verschwommenheit unnötig erhöht.

2 Das „Berliner Programm” gab konservativer Agitation keinen Raum, und wurde von der Kreuzzeitung entsprechend gewertet.

"Karl Brämer, „Die deutsche Arbeiterbewegung unserer Tage”, III, in: Arbeiterfreund, 1864, S. 161ff., gibt eine vernünftige Erklärung, wie es zum Eingreifen von Streckfuss kam: Am 19. Oktober sei der Beschluss gefasst 
Verworfen wird das Prinzip eines Kongresses, ganz unabhängig von der Tagesordnung, denn wie können 300-400 Arbeiter es unternehmen, einen deutschen Arbeiterkongress $\mathrm{zu}$ berufen, und die Berliner Arbeiterschaft zu vertreten, wenn diese nach vielen, vielen Tausenden zählt: „Die Entwicklung der grossen sozialen Frage der Gewerbefreiheit und Freizügigkeit wird durch den von einer verschwindenden Minderheit der Berliner Arbeiter berufenen und beschickten Kongress wahrlich nicht gefördert, sondern eher gehemmt werden!" Stattdessen sollte das Volk bei den Wahlen auch diese hochwichtige Frage scharf ins Auge fassen, denn es sei Sache von Männern der Wissenschaft und des praktischen Lebens, diese Frage in den verschiedenen Parlamenten zur Sprache zu bringen; sie würden "sicherlich dereinst" zum Siege kommen! Wie die Freizügigkeit „durch ganz Deutschland” durch Debatten in den Sonderparlamenten zustandekommen solle, wird nicht klar, aber Streckfuss spricht auch von volkswirtschaftlichen Kongressen, die sich dieser Frage annähmen: ihnen gegenüber wird mit anderem Massstab gemessen als bei den Arbeitern!

Der Kongress sei prinzipiell abzulehnen, aber noch mehr sei er taktisch verfehlt, denn er störe den „geistigen Kampf für die Verfassung”, der mit der Vertagung des Abgeordnetenhauses am 13. Oktober in eine neue Phase getreten war. Der "geistige Kampf" bedeutete den Entschluss, auf rein parlamentarischem Boden zu bleiben. Deswegen durfte es keine Volkserregung geben, durfte keine unkontrollierbares Gremium zusammentreten. Vorgeschoben wurde die Möglichkeit reaktionärer Umtriebe, gemeint war die Furcht in den eigenen Kreisen vor der roten Gefahr: „Der verfassungstreuen Partei werden von ihren Gegnern alle mögliche Sondergelüste, revolutionäre Bestrebungen usw. untergeschoben, man möchte ihre schwankenden Anhänger

worden, 12 Deputierte mit je 15 Talern Diäten auszustatten (der Kongress sollte eine volle Woche dauern). Die Kosten sollten durch freiwillige Beiträge der Arbeiter unter Ausschluss aller Personen anderer Stände aufgebracht werden. „Unzufrieden mit diesen Beschlüssen, schieden mehrere Mitglieder des Ausschusses aus, an deren Stellen neue hineingewählt wurden". Als die demokratische Presse sein Vorgehen getadelt habe, habe Eichler gereizt erklärt, „er habe mit den Autoritäten in gewerblichen Angelegenheiten vorher Rücksprache genommen", und damit "erweckte er ein anscheinend unbegründetes, aber ihm schädliches Misstrauen in seine politische Zuverlässigkeit". Es ging um zwei Dinge: die Selbständigkeit der Kassen, die die Bewegung von den liberalen Gönnern unabhängig machen konnte, und die Festsetzung eines Termins für den Kongress ohne vorhergehende Rücksprache. Wie der dunkle Hinweis auf die "Autoritäten" in "gewerblichen Angelegenheiten" damals verstanden worden ist, lässt sich nicht feststellen. Vielleicht hat Eichler damit seine Gewissheit begründet, die Erlaubnis der sächsischen Regierung - die noch ausstand - werde rechtzeitig eintreffen. Brämers Darstellung beruht auf Zeitungsberichten und persönlicher Erfahrung; er wird zu sehr übersehen. 
wieder schrecken mit den Gespenstern der roten Republik, einer sozialen Arbeiterbewegung, um sie durch solche törichte Furcht in die Reihen der Reaktion hineinzuscheuchen. - Wie dies im Jahre 1848 gelungen ist, so hofft man, dass dies auch heute gelingen werde!" In diesen ängstlichen Kreisen äussere man den unbegründeten Verdacht, dass die ganze Arbeiterbewegung von der Reaktion ausgehe. Wenn die Arbeiter trotz der Mahnung weiter den Kongress betrieben, dann dienten sie unbewusst der Reaktion, weil sie den Feinden der Freiheit scharfe Waffen in die Hände gäben. ${ }^{1}$

Der Bitte des Arbeiterkomitees um Abdruck des Aufrufes wurde nicht Folge geleistet, aber der Streckfuss'sche Artikel wurde durch alle liberalen Zeitungen übernommen, unter anderem durch die Mitteldeutsche Volkszeitung. Er bildete die Grundlage aller weiteren Aussprachen in der Kongressfrage. Wie konnte es auch anders sein, wenn auch diese sächsiche radikale Zeitung den Heldenmut des "geistigen Kampfes" für die preussische Verfassung immer wieder begeistert schilderte und als Vorbild hinstellte? Es sollte nicht wunder nehmen, wenn aus dem unbeabsichtigten, der Reaktion erwiesenen Dienst bald das „bewusste Handwerkzeug” im Dienst Bismarcks wurde. Es ist unbedingt $z \mathfrak{u}$ beachten, dass Streckfuss hier keineswegs gewisse Vorschläge oder Programme, sondern das unzeitgemässe Zusammentreten des Kongresses als Dienst der Reaktion sieht. Erst eine weitere Wende der Ereignisse brachte den Vorwurf in Zusammenhang mit Eichlers Vorschlägen.

Eichler hatte es unternommen, die Leipziger von ihren "verkehrten" Vorstellungen abzubringen, zu diesem Zweck sich nach Leipzig eingeladen, um erst mit dem Komitee und dann mit den Arbeitern Aussprache $\mathrm{zu}$ halten. ${ }^{2}$ Über diese entscheidend wichtige Konferenz besitzen wir kein Dokument, sondern nur Aussagen der Erinnerungsliteratur. Wir wissen nur, was Fritzsche der Arbeiterversammlung vom 30. Oktober laut Meldung der Mitteldeutschen Volkszeitung berichtete, aber das ist beachtlich. Er sagte: „Ein Deputierter aus Berlin sei am 25. Oktober hier gewesen, sei aber dem in der vorigen Versammlung ernannten Komitee, das eine Sitzung mit ihm gehalten hat, wenn auch auf dem Boden der arbeitenden Klassen stehend, doch wie ein Werkzeug der Reaktion vorgekommen, in welchem Sinne sich auch die Berliner Blätter über die leitenden Persönlichkeiten des

2 Siehe S. 357, Anm. 2. 
dortigen Vereins ausgesprochen hätten. Man habe sich gegen einen Kongress nach der von ihm beliebten Weise erklärt..."1

„Werkzeug der Reaktion” im Sinne der „Berliner Blätter” heisst in diesem Zusammenhang einfach, dass Eichler den Kongress zu unpassender Zeit als Störenfried des parlamentarischen Austrags des Verfassungskonflikts anberaumen wollte; sicher nicht, dass er eine Förderung der Arbeiterbewegung durch Bismarck in Vorschlag gebracht habe: Fritzsche hätte das sonst angedeutet. Dazu passt auch seine Mitteilung, der Berliner Abgeordnete habe auf einem baldigen Zusammentreten des Kongresses bestanden, um durch die Resultate desselben auf das Preussische Abgeordnetenhaus einzuwirken. Darin sah Fritzsche allerdings einen Streitpunkt, und er schlug im Namen des Komitees eine Vertagung bis Mitte Januar vor. Das Komitee sollte die Wiederaufnahme der Sitzungen des vertagten Parlaments abwarten, weil es damals schien, als würde durch die Wiederaufnahme der Sitzungen die Krise des Verfassungskonfliktes erreicht werden, falls nicht durch Gewaltmassnahmen der Krone überhaupt eine Bürgerkriegssituation entstehen werden (auf die ja auch Lassalle damals spekulierte). In dieser Lage verlangte Fritzsche, „dass es jetzt vor allen Dingen darauf ankäme, alle Sonderinteressen, deren besondere Betonung dazu dienen würde, einen Teil der liberalen Partei zurückzuschrecken, ruhen zu lassen, und in jeder Beziehung mit der Fortschrittpartei Hand in Hand die gemeinsamen Ziele zu verfolgen", besonders, da mit der Erstrebung der deutschen Grundrechte, wie sie der Nationalverein nunmehr verlange, auch den Sonderinteressen der Arbeiter gedient sei. Eichler habe dagegen verlangt, grade während der Parlamentspause zum Kongress zusammenzutreten, weil „durch die von ihm zu erstrebenden Resultate desselben auf das preussische Abgeordnetenhaus hingewirkt werden solle".

Fritzsche und das Komitee wie auch Eichler weisen auf „Sonderinteressen" der Arbeiter hin; Fritzsche meint die politischen Gleichberechtigungsansprüche, die nicht mehr vordringlich seien, nachdem der Nationalverein sie prinzipiell anerkannt habe, während Eichler soziale Verbesserungen meint und hofft, sie von der liberalen Mehrheit des Abgeordnetenhauses grade wegen deren politischer Zwangslage abtrotzen zu können. Wenn wir nicht von der Agentenrolle Eichlers wüssten, würde seine Taktik einleuchtend scheinen, besonders wenn man bedenkt, dass der Verfassungskonflikt gar nicht zur Entscheidung stand, die Kongressbewegung aber nur Erfolg haben konnte, wenn

1 Bericht der Deutschen Allgemeinen Zeitung, Leipzig, 31. Oktober. Der Bericht ergänzt đie Darstellung der Mitteldeutschen Volkszeitung „Die zweite Arbeiterversammlung", in Nr 256. 
sie sich schlagartig durchsetzte. Vom Standpunkt des Leipziger Komitees aus gesehen, das politisch dachte, war allerdings im Augenblick das Vermeiden eines offenen Konflikts mit der Fortschrittspartei und dem Nationalverein das Gegebene.

Der Beschluss der Leipziger Arbeiterversammlung vom 30. Oktober entsprach natürlich der Berichterstattung Fritzsches, besonders da Eichler seine Auffassung nicht darlegen konnte. Es wurde beschlossen, den Kongress bis Januar zu vertagen, seine Leitung in die Hand zu nehmen und eine Verständigung mit dem Berliner Komitee anzubahnen, damit die Fortschrittspartei ihr Veto zurücknehme. Zu diesem Zweck wurden Fritzsche und Hecht nach Berlin delegiert. ${ }^{1}$

Die geschichtliche Lage der Berliner und Leipziger Bewegung scheint vollkommen klar. Gemeinsam war beiden die Tradition der „Verbrüderung” mit ihren öffentlichen Arbeiterversammlungen, die allein berechtigt seien, verbindliche Beschlüsse im Namen der Arbeiterklasse - oder wie es damals hiess: des Arbeiterstandes - zu fassen: unterschiedlich war die Auffassung des Zieles und der Taktik. Berlin wollte eine von den Liberalen unabhängige rein soziale Bewegung, die bereit war, Verbesserungen anzunehmen, wo sie sich boten, während die Leipziger am linken Flügel des Nationalvereins und der Fortschrittspartei, wenn auch als konstituierte Partei, ihren politischen Kampf führen wollten. Dass in der Berliner Sicht Platz für manche Spielart eines Zusammengehens mit der Reaktion war, liegt auf der Hand, nur waren die Dinge durchaus nicht so geklärt wie sie uns in der Rückschau scheinen. Die Berliner Fortschrittsführer hatten instinktmässig erfasst, dass Eichler auf jeden Fall gefährlich war, auch wenn sie vor der Hand keinerlei Beweise für seine Verbindung mit der Regierung hatten und eine solche zunächst nicht einmal annahmen.

Dieser Darstellung steht allerdings Bebels persönliche Erinnerung entgegen, wenn er in seinem Werk Aus meinem Leben schreibt:

„Anfangs Oktober kam als Berliner Vertreter der Maler und Lackierer Eichler nach Leipzig zu einer Besprechung, der auch ich als Mitglied des Komitees beiwohnte. Diese Besprechung fand in der Restauration Zum Joachimstal in der Hainstrasse statt. Eichler ging gleich aufs Ganze. Er führte aus, dass die

1 Berliner Volkszeitung, $\mathrm{Nr} 254$ (30. Oktober): Die Meldung ist nach der Besprechung, aber vor der beschlussfähigen Versammlung verfasst: „Nachdem Herr Eichler in Leipzig mit dem dortigen Arbeiterkomitee persönlich Rücksprache genommen, ist, wie uns mitgeteilt wird, der projektierte Arbeiterkongress bis zum Anfang Januar 1863 vertagt worden." 
Arbeiter von der Fortschrittspartei und dem Nationalverein nichts zu erwarten hätten. Die Mehrzahl der Komiteemitglieder teilte auf Grund der gemachten Erfahrungen diese Ansicht. Weiter fuhr Eichler fort: er habe die Gewissheit - und damit entpuppte er sich nach unserer Ansicht als Agent Bismarcks -, dass Bismarck für die Einführung des allgemeinen, gleichen und direkten Wahlrechts zu haben sei, und auch bereit wäre, die nötigen Mittel (60.000 bis 80.000 Taler) zur Gründung einer Produktivgenossenschaft der Maschinenbauer herzugeben."1

An diesem Bericht stimmt nicht allein das Datum nicht. Völlig unmöglich ist, dass damals das Verhältnis zum Nationalverein und zur Fortschrittspartei in der Art zur Sprache gekommen sein könnte, wie es hier dargestellt wird. Die Leipziger verlangten wiederholt ein Zusammengehen mit „Fortschritt” und Nationalverein selbst gegen deren Willen, aber das immer erklärte Ziel war die aktive Mitarbeit am Nationalverein. Dementsprechend hatte Fritzsche sich in der Arbeiterversammlung ausgesprochen. Wenn nun Bebel diesen Kardinalpunkt der damaligen Entwicklung falsch darstellt, so muss auch die Erwähnung Bismarcks in der Form, in der es hier geschieht, als unwahrscheinlich in Frage gestellt werden: grade weil Eichler Agent Bismarcks war, musste er jeden Verdacht in dieser Hinsicht vermeiden; die Frage der Oktroyierung des Wahlrechts durch Bismarck war noch gar nicht aufgetaucht und nur die Unterstützung von Produktivgenossenschaften durch Staatskredit könnte vielleicht in der Besprechung zur Sprache gekommen sein, obwohl auch das nicht warscheinlich ist. Es ist nicht schwer zu verstehen, wieso Bebel dieser Gedächtnisfehler unterlaufen konnte. Durch Lassalles Agitation wurden im Frühjahr 1863 die genannten Fragen aktuell, und damals kam allerdings in die Zeitungen, dass Eichler Vorschläge für Finanzierung einer Kooperativgenossenschaft für den Bau von Eisenbahnwagen gemacht habe, die aber unbeantwortet geblieben seien. Ausserdem stimmt es, dass kurze Zeit nach Eichlers Auftreten behauptet wurde, das Leipziger Komitee habe Beweise für die Agententätigkeit Eichlers in den Händen. Diese Beweise wurden nie beigebracht und der mögliche Inhalt solcher Beweise einfach interpoliert.

Die Verhandlungen der zweiten Leipziger Arbeiterversammlung standen völlig im Zeichen der Streckfusschen Ideen und wahrscheinlich der Instruktionen, die von Berlin an die Redaktion der Mitteldeutschen Volkszeitung gelangt waren; daher der Vorschlag, Leipziger Delegierte nach Berlin zur Übernahme der Bewegung zu senden, die

1 August Bebel, a.a.O., S. 62. 
„zur Kenntnisnahme der Verhältnisse aber womöglich etwas eher abreisen sollten", was dann zu der Vorbesprechung im Hause des v. Unruh führte. Dass das Arbeiterkomitee Leipzigs ein Werkzeug in den Händen der Fortschrittspartei zur Beseitigung einer unbequemen Bewegung in Berlin geworden war, ist offensichtlich. Damit war ein Hindernis des „geistigen Kampfes für die Verfassung” beseitigt.

Die Arbeiterversammlung machte sich das „Berliner Programm” zu eigen, strich aber auf Vorschlag Hartwigs und Dolges den Punkt der Vorbereitung einer Weltausstellung. Den beiden Deputierten Vahlteich und Hecht wurde der Auftrag gegeben, bei den Berliner Arbeitern dahin zu wirken, „dass auch sie der Fortschrittspartei auf das engste sich anschliessen möchten", in der Hoffnung, "dass die Fortschrittspartei nicht fernerhin auf weitere Vertagung der ganzen Bewegung hinwirke". Dass soviel demonstrative Anhänglichkeit an die Fortschrittspartei ihren Entschluss, den Kongress zu vereiteln, doch nicht änderte, scheint zunächst auffallend, erklärt sich aber leicht, wenn man beachtet, dass die organisatorischen Vorschläge, die zur Annahme kamen, das demokratische Versammlungsprinzip zur Herrschaft brachten, nachdem die Anträge abgelehnt worden waren, das Arbeiterkomitee statt aus direkten Wahlen der freien Versammlungen aus Vertretern der verschiedenen Berufszweige erstehen zu lassen. An einer Konstituierung der Arbeiterklasse konnte den Fortschrittsführern nicht gelegen sein. Diejenigen Initiatoren der Versammlungsbewegung, die sich mehr als Vertreter ihrer Berufe als der Klasse fühlten, schieden aus dem definitiven Komitee aus, so auch Hecht, der ursprünglich nach Berlin reisen sollte. Seine Stelle nahm Fritzsche ein. Interessant ist, dass auch Bebel dem neuen Komitee nicht beitrat. ${ }^{1}$

Die angehenden Arbeiterführer Fritzsche und Vahlteich liessen sich dazu missbrauchen, die den Liberalen ungelegene Arbeiter-

1 Das Komitee, das zur ersten Arbeiterversammlung aufrief, war so zusammengesetzt, dass die verschiedenen Handwerke vertreten waren (Mitteldeutsche Volkszeitung, Nr 227), das zweite war eine Gesamtvertretung (ebda, Nr 256). Nach der endgültigen Konstituierung wurde der Brauch aufgegeben, jedes Komiteemitglied mit Bezeichnung des Standes unterzeichnen zu lassen, und Vahlteich unterzeichnete als Vorsitzender allein. Das könnte Zufall sein, wenn nicht die Debatte über die Vertretung von Korporationen vorangegangen wäre. Die Tatsache, dass das Komitee sich als Gesamtvertretung der Leipziger Arbeiter ansah, wiegt schwerer als die Tatsache, dass nach dem Ausscheiden einiger Handwerker, Nicht-Arbeiter, wie der Fabrikant Dolge, in das Komitee eintraten: das waren erprobte Demokraten und ehemalige Handarbeiter, die sich hochgearbeitet hatten. Das Ausscheiden Bebels bei dieser Gelegenheit ist für seine damalige Entwicklung charakteristisch. 
bewegung mit Hilfe der sehr undemokratischen Praktiken der Vorkonferenz zu vereiteln. Das ist durchaus entschuldbar, wenn man bedenkt, dass erfahrene Volksmänner und Parlamentarier es mit jungen und unerfahrenen Menschen zu tun hatten. Auffällig dagegen ist, dass sie auch in der Rückschau der Erinnerungsliteratur den Missbrauch nicht erkannt haben, sondern auf ihre Leistung sehr stolz waren, hatten sie doch im Hause Unruhs über demokratisches Stimmrecht und Unterstützung der Kongressbewegung durch die Fortschrittspartei verhandelt. ${ }^{1}$ Sie haben nicht gemerkt, dass man schon in der darauf folgenden öffentlichen Versammlung den Kongress auf die lange Bank schob; als sie es merkten, waren sie ratlos und haben sich bedingungslos Lassalle als Retter aus der Not in die Hand gegeben. Nur eins haben sie erreicht: die Fortschrittspartei wagte es nicht, Eichler durch irgendeine liberale Grösse $z u$ ersetzen: soviel Respekt hatten sie doch vor dem Emanzipationsstreben der Arbeiter bekommen. Sie bestimmten den ehrlichen und radikalen Schriftsetzer Dittmann, einen bewährten 48-er, zum Vorsitzenden des Berliner Komitees: sie sollten es noch oft bereuen. ${ }^{2}$

${ }^{1}$ Julius Vahlteich hat in seiner letzten Lebenszeit in Amerika verschiedentlich zur Erinnerungsliteratur beigetragen, indem er ganze Abschnitte von einem Aufsatz in den anderen übertrug. Der Sinn ist immer der gleiche: Die Bewegung ging von Leipzig aus; dort wurde das Initiativkomitee gewählt und das Programm aufgestellt; die Liberalen waren die Düpierten: „Wir wollten Organisation iiber das ganze Land und konnten diese doch nicht schaffen, ohne die Hilfe der liberalen Presse, die sich sofort feindlich gegen uns stellen musste, wenn wir die sozialistische Fahne entrollt hätten. Wir planten also einen deutschen Arbeitertag und empfahlen eine Tagesordnung, die das Bürgertum nicht vor den Kopf stossen konnte. Freizügigkeit und Gewerbefreiheit, die Schlagworte der Zeit, waren das Aushängeschild, Organisation zur Vertretung der Arbeiterinteressen war das Ziel. Wir liessen in einer Volksversammlung ein ,Zentralkomitee zur Einberufung eines deutschen Arbeitertages' wählen. [...] Die Berliner Fortschrittler waren ziemlich ratlos. Sie wussten nicht, was sie aus uns machen sollten, die Arbeiterbewegung störte ihre Kreise und war ihnen höchst unangenehm. Wir bestanden aber fest auf einer gesonderten Arbeiterorganisation und man einigte sich dahin, in der Volksversammlung für die Einberufung des Arbeitertages auf Ende Januar 1863 nach Leipzig einzutreten [...] So wurde denn auch von der riesig besuchten Versammlung beschlossen." 2 Darüber: Bernstein, a.a.O., S. 118f. Aufschlussreich ist die Statutenänderung des Berliner Arbeitervereins, der die Aufgaben der öffentlichen Arbeiterversammlung übernahm, wie Schulze es gefordert hatte. Im Februar heisst es: „Unter dem Namen ,Arbeiterverein' hat sich für Berlin ein Verein gebildet, der die Förderung der geistigen und materiellen Interessen seiner Mitglieder anstrebt und sich namentlich die Durchführung des von den Berliner Arbeitern aufgestellten Programms zur Beschickung eines Kongresses zur Aufgabe stellt." In der Fassung vom Juli 1863 heisst es nach Auslassung des inzwischen hinfälligen Kongresses u.a. in § 2, Absatz 3: „Der Verein will jede Sonderstellung des Arbeiters beseitigen" - ein Lieblingsgedanke Dittmanns, der Arbeitsbücher und sonstige Sonderbeschränkungen für Arbeiter bekämpfte. 
Abgesehen von einer erheblichen Opposition seitens der Eichlerschen Fraktion, entsprach die glanzvolle Massenversammlung vom 2. November 1862 ganz den Erwartungen; das Leipziger Komitee wurde zum Zentralkomitee zur Vorbereitung eines allgemeinen deutschen Arbeiterkongresses für ganz Deutschland erklärt, und somit die Konstituierung der deutschen Arbeiterklasse von Seiten der Liberalen geduldet: Streckfuss nahm in einer gewundenen Erklärung einige anstössige Sätze zurück, und Schulze kleidete seine Gegnerschaft in Sätze ein, die wie Zustimmung klangen, nur vor „Ubereilung” warnte er offen.

Das Berliner Komitee wurde zum Rang eines Lokalkomitees herabgedrückt, womit der Hauptzweck der Liberalen erreicht wurde. Der Stärke der Eichlerschen Opposition wurde darin Rechnung getragen, dass auch Eichler dem Lokalkomitee angehören sollte. ${ }^{1}$ Eichlers Rolle als Arbeiterführer war damit ausgespielt, und die Arbeiterbewegung war in eine neue Phase ihrer Konstituierung getreten, belastet allerdings mit der Konferenz im Hause Unruhs.

\section{VI}

Von Anschuldigungen gegen Eichler seitens der Leipziger Delegierten ist auch in den ausführlichsten, teilweise sogar stenographischen Berichten nichts zu lesen; sie sind sicherlich nicht öffentlich erhoben worden, denn sonst hätte die Wiederwahl Eichlers nicht erfolgen können, aber Gerüchte wurden in Umlauf gesetzt, wie die Berichterstattung des Arbeitgeber beweist. Nachdem er Eichler als Störenfried der Londoner Expedition, als „eitlen, ehrgeizigen Charakter" verurteilt und Schulzes Rolle als Retter der Bewegung hervorgehoben hatte, fährt er fort: „Die letztere Forderung [dass die Arbeiter sich von der Politik fernhalten sollten], verbunden mit dem eigentümlichen Auftreten Eichlers, gab in Leipzig sogar Anlass zu dem Verdachte, Eichler sei ein Werkzeug der Reaktion, beauftragt die preussischen Arbeiter von der jetzt so ernsten wichtigen politischen Bewegung in Preussen abzuziehen. Man ist aber damit offenbar zu weit gegangen. Eichler ist nichts weiter als ein ehrgeiziger Intrigant, der schon 1848 und auch jetzt nur aus Eitelkeit sich der ganzen Bewegung

17 Mitglieder des alten Komitees wurden in das neue Lokalkomitee übernommen. Ein Jacobsohn hatte sich in der Versammlung gegen die Bevormundung durch die "Gelehrten" ausgesprochen und kam nicht ins neue Komitee; er war vermutlich Jude und daher wohl kaum, ,reaktionär. Die Aufsässigkeit der Berliner Arbeiter, weniger Umtriebe der Reaktion, waren die Zielscheibe des fortschrittlichen Angriffs. Dem Dittmann, der bei der Berliner Reform mitarbeitete, wurde Hoffmann als Mann der Berliner Volkszeitung beigegeben. 
angeschlossen hat."1 Die Rheinische Zeitung dagegen weiss am 6. November nach einem Bericht aus Leipzig vom 3. November zu berichten: „Einige Tage vorher war jemand aus Berlin bei dem Komitee der Leipziger Arbeiter gewesen, um zu versuchen, im Interesse des ,Preussischen Volksvereins' Beziehungen anzuknüpfen. Diesen Emissär, der selbst dem Arbeiterstand angehört, hatte das Komittee durchschaut und also abgewiesen."

Es ergibt sich also das paradoxale Bild, dass Eichler in Berlin selbst weiter als Komiteemitglied wirkt, in Leipzig aber als überführtes Werkzeug der Reaktion, in Frankfurt einfach als Charlatan und in Düsseldorf als Emissär des „Volksvereins” gilt. Nur an Bismarck dachte man damals in diesem Zusammenhang noch nicht. ${ }^{2}$ Eichler hat auf diese Meldung mit einer Erklärung in der Berliner Volkszeitung geantwortet, zu deren Abdruck in allen liberalen Zeitungen er aufforderte und zugleich auf seine Arbeitsentlassung angespielt. „Verschiedene Zeitungen bringen seit einiger Zeit Berichte und Artikel, nach deren Inhalt man annehmen könnte, als ob ich unter dem Einflusse der Reaktionspartei die jetzige Arbeiterbewegung hervorgerufen und bisher geleitet hätte, ja, in einem aus Leipzig datierten Bericht ist sogar offen gesagt, ich wäre dem Leipziger Komitee als ein ,Werkzeug der Reaktion' erschienen." Er beteuert ein erklärter Gegner der reaktionären Parteien und ein alter Vorkämpfer der liberalen zu sein, so dass ihn jener Vorwurf schmerze: „Gegen die Bezeichnung ,Werkzeug der Reaktion' protestiere ich ganz entschieden und überlasse das Urteil hierüber meinen Kameraden und allen denen, die mich näher kennen." Eichler erklärt dann weiter, dass er zu seinem Bedauern nun weniger tätig sein könne, fordert aber auf, einig zu bleiben, um die Bestrebungen der Gegner zu Schanden zu machen.

Der Kreis ist damit geschlossen. Von Berlin aus hat man Leipzig gewarnt, die ganze dortige Bewegung sei eine Mache der Reaktion, worauf Leipzig den Verdacht ausspricht, der Berliner Delegierte sei ein Werkzeug der Reaktion, worauf Berlin auf Nachricht von Leipzig hin die Tatsache kundtut, in Leipzig habe man den Eichler als Werkzeug der Reaktion entlarvt. ${ }^{3} \mathrm{Da}$ die Leipziger jeden Beweis für ihren Verdacht schuldig blieben, wurde die Lücke beliebig und in charakteristischer Weise ausgefüllt. Max Wirth rächte sich nun an der Volkszeitung, die seinerzeit den Nörgeleien Eichlers Platz gegeben hatte; die ehemaligen Kommunisten Becker und Bürgers in der radikal-liberalen Rheinischen Zeitung wandelten das „Werkzeug der

\footnotetext{
1 Arbeitgeber, Beilage zu Nr 304, „Der Arbeitertag”, 11. November 1862.

2 Rheinische Zeitung, Nr 338, 6. November 1862.

${ }^{3}$ Berliner Volkszeitung, 7. November 1862.
} 
Reaktion” stilgerecht in einen „Emissär” um, der versucht habe, Verbindungen anzuknüpfen. Wer nicht wusste, was uns heute aktenmässig bekannt ist, und die liberalen Intrigen gegen die Arbeiterbewegung durchschaute, musste - wie Liebknecht - glauben, hier sei im Interesse der Hintertreibung der Klassenorganisation Unrecht geschehen.

In diesem Zusammenhang ist es wichtig, festzustellen, dass sich Wageners Kreuzzeitung zur Kongressbewegung ziemlich zurückhaltend verhielt. Meistens begnügte sie sich, Berichten der liberalen Zeitungen hämische Bemerkungen anzuhängen: der vorgeschlagene Kongress sei ein „demokratischer blauer Montag"; die Liberalen seien durch die soziale Bewegung in grosse Verlegenheit geraten, weshalb sie versuchten, die Bewegung zu gängeln; werde ihnen ein Arbeiter unbequem, so massregelten sie ihn. Die Arbeiterbewegung sei ein Werk der "Maulwürfe" von 1848-49, um das Abgeordnetenhaus souverän zu machen. Eichlers Protest kommentierte sie mit der Bermerkung: „Die konservative Partei kann die Männer nur bedauern, die - obschon ihre politischen Gegner - doch für ihr Streben solche Erfahrungen machen müssen." Die Frage, ob trotz dieser Haltung die Zeitung von Eichlers Diensten für Bismarck wusste, lässt sich nicht beantworten; sie mag jedoch ohne weitere Kenntnis in Eichlers Richtung einen günstigen Anhaltspunkt für ihre Bestrebungen gesehen haben. ${ }^{1}$

Auf Eichlers Protest gegen die Verdächtigungen, die die Leipziger Delegierten hinter seinem Rücken verbreiteten, statt ihn in öffentlicher Rede anzuklagen, antworteten die liberalen Blätter, dass ,in den nächsten Tagen eine offizielle Erklärung erscheinen werde, welche die nötigen Beweise für die Doppelrolle beibringen wird, welche der Arbeiter C. Eichler gespielt hat." Eine solche Erklärung war auch deshalb geboten, weil Dittmann als Vorsitzender des Komitees in Leipzig angefragt hatte, worauf sich ihre Anschuldigungen eigentlich stützten, und er ohne wirkliche Beweise Eichler weiter als Ehrenmann behandeln müsse. ${ }^{2}$ Zudem war ein Inserat in der Volkszeitung erschienen, das eine öffentliche Sammlung zu Gunsten Eichlers ankündigte, falls Beweise für die Anschuldigungen nicht beigebracht würden. ${ }^{3}$ Aber noch Ende Dezember standen die Beweise aus, und in der Ar-

1 Wageners sehr persönliche Ansichten über die soziale Frage werden zu sehr den Konservativen im allgemeinen zugeschrieben. Die Kreuzzeitung (Neue Preussische Zeitung) war selbst Huber gegenüber sehr zurückhaltend. Der Hauptton ist Schadenfreude, z.B. Kreuzzeitung vom 8 . November über Eichlers Massregelung; Leipzig, 15. November, "Zum Arbeitertag".

2 Der Brief Dittmanns im IISG, Bestand ADAV/Hatzfeldt.

3 Berliner Volkszeitung, Nr 304, 30. Dezember 1862. 
beiterversammlung vom 28. Dezember liess Dittmann die Korrespondenz mit dem Leipziger Komitee verlesen, aus der hervorgeht, dass die „Antwort des Komitees keine bestimmten Tatsachen gegen Eichler aufstellt, aber aus seinen Ausführungen den Verdacht geschöpft haben will, von dem es sich auch jetzt nicht freimachen könne". Dittmann fügt ausdrücklich hinzu, dass er die Beschuldigungen gegen Eichler auch jetzt nicht glaube. Wenn es überhaupt Wege gibt, das Nichtvorhandensein von etwas zu beweisen, so wohl das Fehlen der Unterlagen für die Leipziger Behauptungen; es mochte noch Sinn haben, in einer öffentlichen Versammlung mit den Beweisen zurückzuhalten, aber es fehlte jeder Grund, den Berliner Kollegen, mit denen man zusammenarbeiten musste, die Beweise vorzuenthalten: sie existierten einfach nicht.

Schulze hatte verlangt, von öffentlichen Massenversammlungen in Berlin abzusehen, und die Aufklärungsarbeit der Berliner Arbeiter im Rahmen eines festen Arbeitervereins zu betreiben. Eichler widersetzte sich im Laufe langwieriger Diskussionen dieser Änderung, drang aber nicht durch und hielt sich einige Zeit den Verhandlungen fern; im Laufe des Jahres 1863 tauchte er immer wieder auf, insbesondere versuchte er die Lassallesche Agitation zu benutzen. Sein Auftreten in der berühmt gewordenen Versammlung am 19. April hat Lassalle erheblichen Schaden zugefügt. Damals wurde es immer klarer, dass hier ein bestimmtes Interesse vorliegen müsse, auch wenn sich der Verdacht nicht direkt belegen liess. Jetzt wurde eine Verbindung Eichlers zu Bismarck plausibel, und die Rheinische Zeitung konnte sie indirekt belegen, indem sie darauf hinwies, der Handelsminister Graf Itzenplitz habe ein Experiment abgelehnt, dass der Innenminister Graf von Eulenburg befürwortet habe. Es handele sich um Eichlers Projekt der Gründung einer Wohnkolonie am Plötzensee. Wie es scheint, liess der freihändlerische Minister die Nachricht an die Redaktion der Rheinischen Zeitung gelangen, um das Projekt $\mathrm{zu}$ torpedieren. ${ }^{1}$

Diese sensationelle Meldung machte die Runde durch die liberalen Zeitungen, und bewegte Eichler dazu, seine der Regierung gemachten Vorschläge bekannt zu machen - Vorschläge, deren Spuren sich in den Akten finden. Er beendigte seine Erklärung mit dem Satz: „Da ich nun seit heute weder Antwort erhalten, noch überhaupt über den Verbleib meiner Entwürfe irgend etwas weiss, so sind alle über meine Person dieserhalb gebrachten Notizen als völlig unwahr zu bezeichnen und behalte ich mir die Veröffentlichung dieser Entwürfe vor, um sie dem Urteil des Publikums preiszugeben."2

1 Berliner Volkszeitung, Nr 88, 16. April 1863.

2 Ebda, Nr 89, 17. April 1863. 
Der Arbeitgeber weiss eine Woche später die Gründe darüber anzugeben, warum Eichler keinen Bescheid erhielt, eine Meldung, die sicherlich sensationell ist: „Der Lackierer Eichler von Berlin hatte, wie neulich berichtet, die Absicht, eine Arbeiterkolonie am Plötzensee anzulegen, und dem Ministerium einen Plan dazu vorgelegt. Herr v. Bismarck hatte den Plan genehmigt und eine Unterstützung aus Staatsmitteln zugesagt. Der Handelsminister, Graf Itzenplitz, hat sich nun neuer Zeit um die genossenschaftliche Bewegung und die Grundsätze Schulzes, den er bloss als politischen Agitator kannte, gekümmert und nach den Berichten, die er sich von den Ministerialbeamten darüber geben liess, eine wohlgefällige Meinung über die Genossenschaften bekommen. In deren Folge veranlasste er im Ministerium eine Zurücknahme der dem Eichler gemachten Zusagen und eine günstigere Stellung gegenüber den Genossenschaften."1

Es ist also Schulze gelungen, sein Prinzip der "Selbsthilfe" durch Genossenschaften dem Handelsminister nahezulegen und die sozialkonservativen Pläne zu durchkreuzen. Hier wird zum ersten Mal Bismarck in begründete Verbindung mit Eichler gebracht, und hier dürfte die Quelle der Bebelschen und Vahlteichschen Behauptungen sein, die anachronistisch mit dem Auftreten Eichlers in Leipzig ein halbes Jahr vorher verbunden wurden. Schulzes Initiative hängt natürlich mit dem Erscheinen des "Offenen Antwortschreibens" zusammen, in dem die "Staatshilfe" propagiert worden war. Dass Schulze die Staatshilfe mit Hilfe eines Staatsministers verdrängt hatte, galt ihm trotz des Verfassungskampfes als unbedenklich. Die Meinungsverschiedenheiten innerhalb der Regierung selbst erklären die Einladung, die einen Monat später an Lassalle erging, sich über Staatshilfe auszusprechen.

\section{VII}

Der Fall Eichler wäre soweit unter den verschiedenen Gesichtspunkten geklärt. Er beweist, wie schwierig der Boden des „deutschen Paris” für das Wachsen einer wahrhaft demokratischen und selbstbewussten Arbeiterbewegung war; wie vorausschauend Bismarck die Hantierung der sozialen Frage grade in der Hauptstadt versucht hat, ${ }^{2}$ und wie argwöhnisch das liberale Bürgertum seine Position dort zu behaupten suchte. Eichler hatte Anhänger, die, soweit sich erkennen lässt, in ihm einen echten Arbeitervertreter sahen, sich von der liberalen Hono-

1 Arbeitgeber, Spalte 3009.

2 Die Überbetonung Bismarcks, wie unmöglich es ihm gewesen sei, bei Antritt seiner Tätigkeit im Ministerium sich mit Dingen zu befassen, die nicht direkt mit den dringendsten Geschäften im Zusammenhang standen, ist auffällig, 
ratiorenpolitik abwandten und ihr soziales Interesse direkt zu vertreten suchten. Die Verketzerung dieser Opposition als Dienst der Reaktion war im voraus gegeben und nicht von der nunmehr erwiesenen Agententätigkeit Eichlers abhängig. Es gibt zu denken, dass Eichler immer wieder Verteidiger fand, solange er seine Begünstigung der Reaktion abstritt und keinerlei wirkliche Anhaltspunkte für die Verdächtigungen beigebracht wurden. Anders nach dem 16. April, nachdem Eichler seine Projekte bekannt gegeben hatte und diese mit Bismarck in Zusammenhang gebracht wurden: in der grossen Arbeiterversammlung vom 19. April kam er nicht mehr zu Wort und wurde mit den Rufen „Haut ihn!” abgewiesen.

Die historische Rolle der Berliner Kongressbewegung ist mit der Entlarvung Eichlers längst noch nicht abgetan, und selbst Eichlers kurzfristige Tätigkeit ist nicht einfach zu negieren. Umgekehrt ist das Vorgehen der Leipziger im Bunde mit den Berliner Fortschrittlern nicht ohne weiteres zu rechtfertigen, nur weil Eichler eben tatsächlich Agent Bismarcks war. Die Leipziger Kongressbewegung ist verkrüppelt geboren worden, weil sie mit den zweideutigen Praktiken der Fortschrittsführer belastet war und Lassalle ihren Führern nur mit sehr einseitigem Rat zur Seite stand. Zunächst einmal ist die Kongressbewegung unterlegen und die Geburt des ADAV ist dafür kein genügender Ersatz. Die Konferenz im Hause Unruhs hat die Arbeiterbewegung Berlins dem Einfluss von Schulze-Delitzsch ausgeliefert, und der hat ihre gesamtdeutsche Konstituierung systematisch hintertrieben. Aber auch er kam nicht ans Ziel. Um den Einfluss der Leipziger Kongressbewegung lahmzulegen und wenig später, um Lassalle entgegenzutreten, musste er Massenversammlungen von Arbeitern veranstalten; er hat damit die Konstituierung der Arbeiterbewegung doch vorangetrieben.

Schwer abzuschätzen ist der Gewinn Bismarcks. Eichler hat Bismarck sicherlich informiert, aber ob er soweit gekommen ist, „Aufträge" erfüllen zu können, scheint zweifelhaft. Weder das Projekt der Wohnkolonie noch der Wagenbaugenossenschaft sind herangereift ; in der Gesetzgebung ist nichts vorangekommen ausser der Koalitionsfreiheit, die im Jahre 1865 verhandelt worden ist, und die keinerlei „,ständische” Züge aufwiess. Lassalles Propaganda der sozialen Monarchie hat keine Früchte gezeitigt ausser der kurzlebigen Weber-

und die Gelegenheit, die ihm der kleine Gedächtnisfehler Bebels bot, hat Bismarck so übertrieben ausgenutzt, dass die einfachste Erklärung wäre, er hätte durch persönliche Einsicht in die Akten oder durch Referat anderer, vielleicht Buchers, sich wieder in Erinnerung gebracht, dass er längst von Eichler und den Arbeiterversammlungen wusste und sich seinerzeit für deren Verlauf interessiert hatte. 
assoziation in Wüste-Giersdorf. Bismarcks Interesse an der sozialen Frage war zu sporadisch, um gegen die Reibungen innerhalb der Regierungsmaschinerie anzukommen. Weitergehenden phantastischen Plänen hat Bismarck sich nie hingegeben, wie Lassalle und Hermann Wagener erfahren mussten.

Der Berliner Arbeiterbewegung standen grössere Hindernisse entgegen als in den meisten organisch gewachsenen Städten, denn die Arbeiterbevölkerung hatte wegen des ständigen Zuzugs und der räumlichen Zerstreuung wenig inneren Halt. Krisen wie die, die Schweitzer, Dühring, Hasselmann, Most oder Stöcker heraufbeschworen, sind wahrscheinlich mehr auf organische Schwierigkeiten zurückzuführen, als auf Korrumpierung. Das scheint auch für die Eichlersche Phase zu gelten. 